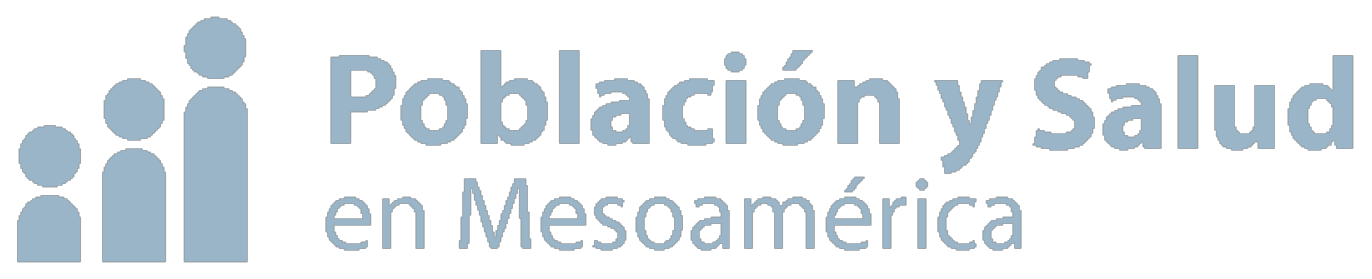

\title{
Retorno social de la inversión tras eliminar la transmisión vertical de chagas, VIH/sida, HB y sífilis: caso de un municipio de la Provincia de Buenos Aires
}

Malena Monteverde, Constanza Silvestrini, Ana Cristina Pereiro, Tamara Wolovich, Mariana Ceriotto y Juan Manuel Castelli.

\section{Cómo citar este artículo:}

Monteverde, M., Silvestrini, C., Pereiro, A. C., Wolovich, T., Ceriotto, M. y Castelli, J. M. (2022). Retorno social de la inversión tras eliminar la transmisión vertical de chagas, VIH/sida, HB y sífilis: caso de un municipio de la Provincia de Buenos Aires. Población y Salud en Mesoamérica, 19(2). Doi: 10.15517/psm.v0i19.46466

\section{(1) $\odot(0$}

ISSN-1659-0201 http://ccp.ucr.ac.cr/revista/

Revista electrónica semestral Centro Centroamericano de Población Universidad de Costa Rica 


\section{Retorno social de la inversión tras eliminar la transmisión vertical de chagas, VIH/sida, HB y sífilis: caso de un municipio de la Provincia de Buenos Aires \\ Social Return on the investment of the elimination of the vertical transmission of Chagas, HIV / AIDS, HB and Syphilis: Case of a Municipality of the Province of Buenos Aires
Malena Monteverde ${ }^{1}$, Constanza Silvestrini ${ }^{2}$, Ana Cristina Pereiro ${ }^{3}$, Tamara Wolovich ${ }^{4}$, Mariana Ceriotto ${ }^{5}$ y Juan Manuel Castelli ${ }^{6}$.

Resumen: Introducción: se busca cuantificar los retornos de la inversión asociados a una intervención en el sistema público de salud de un municipio de la Provincia de Buenos Aires, Argentina, consistente en el fortalecimiento de la estrategia denominada Eliminación de la Transmisión Maternoinfantil de la Infección por VIH, Sífilis, enfermedad de Chagas Congénita e Infección Perinatal por Hepatitis B (ETMI-PLUS). Metodología: el estudio (cuantitativo) se basa en la metodología de Retorno Social de la Inversión (RSI). Se establecieron definiciones ad-hoc para la medición de los retornos sobre la base de los datos disponibles provenientes de diversas fuentes: información primaria de la Secretaría de Salud del MAB; tasas de transmisión congénita de cada enfermedad notificados al Sistema Nacional de Vigilancia de Salud; presupuestos detallados de los recursos asignados al proyecto por parte de la Fundación Mundo Sano y costos de tratamientos e insumos de nomencladores oficiales. Resultados: por cada peso invertido para reforzar la ETMI-PLUS en el MAB, se obtuvo un retorno de casi 4 pesos, gracias a las mejoras en la eliminación vertical de las cuatro enfermedades y al descenso de las complicaciones cardiacas en las mujeres embarazadas diagnosticadas con chagas y tratadas oportunamente. Conclusiones: estos resultados sugieren la existencia de una relación retorno-inversión favorable, analizada bajo una perspectiva conservadora, ya que, se incluyen exclusivamente los ahorros para el sistema de salud y se excluyen otras dimensiones de los retornos vinculadas con las mejoras en los resultados alcanzados.

Palabras claves: enfermedad de Chagas, VIH, hepatitis $B$, sífilis congénita, transmisión vertical de enfermedad infecciosa.

Abstract: Introduction: we seek to quantify the returns on investment associated with an intervention in the public health system of a Municipality of the Province of Buenos Aires, Argentina. This intervention consists of

\footnotetext{
${ }^{1}$ Centro de Investigaciones y Estudios sobre Cultura y Sociedad. Universidad Nacional de Córdoba - Consejo Nacional de Investigaciones Científicas y Técnicas. Córdoba, ARGENTINA. Correo electrónico: montemale@yahoo.com.ORCID: https://orcid.org/0000-0002-5694-5375

2 Facultad de Ciencias Médicas. Universidad Nacional La Plata y Fundación QUANT. La Plata, Provincia de Buenos Aires. ARGENTINA. Correo electrónico: constanzasilvestrini@gmail.com. ORCID: https://orcid.org/0000-0001-8954-0991

3 Fundación Mundo Sano. Ciudad Autónoma de Buenos Aires, ARGENTINA. Correo electrónico: apereiro@mundosano.org ORCID: https://orcid.org/0000-0002-5207-0284

${ }^{4}$ Centro Municipal de Diagnóstico y Prevención de las Enfermedades, Municipio de Almirante Brown. Provincia de Buenos Aires, ARGENTINA. Correo electrónico: tamawolovich@gmail.com_ORCID: https://orcid.org/0000-0002-6462-7502

5 Ministerio de Salud de la Provincia de Buenos Aires. Mercedes, Provincia de Buenos Aires, ARGENTINA. Correo electrónico: mceriotto@gmail.com._ORCID: https://orcid.org/0000-0003-2323-6828

${ }^{6}$ Subsecretaría de Planificación, Prevención y Promoción de la Salud, Municipio de Almirante Brown. Provincia de Buenos Aires, ARGENTINA. Correo electrónico: juanmacastelli@gmail.com_ORCID: https://orcid.org/0000-0003-2177-8017
} 
strengthening the strategy for the Elimination of Mother-to-Child Transmission of HIV Infection, Syphilis, Congenital Chagas Disease and Perinatal Hepatitis B Infection, a strategy called ETMI-PLUS. Methodology: the study (quantitative) is based on the Social Return on Investment (RSI) methodology. Ad-hoc definitions are established for the measurement of returns based on the information available from various sources: primary information from the Ministry of Health of the MAB; rates of congenital transmission of each disease reported to the National Health Surveillance System; detailed budgets of the resources assigned to the project by Fundación Mundo Sano and costs of treatments and supplies from official nomenclators. Results: for each argentinean peso invested in strengthening the ETMI-PLUS in the MAB, a return of almost 4 pesos would have been obtained thanks to the improvements in the vertical elimination of the 4 diseases and the reduction of cardiac complications in pregnant women. Conclusions: these results suggest the existence of a return / investment relationship favorable to the intervention, analyzed under a conservative analysis since savings for the health system are exclusively included and other dimensions of returns associated with improvements in results are excluded.

Key words: Social Health Inequity, Health Status Disparities, Costa Rica, Life expectancy, Violent death.

Recibido: 30 mar, 2021 | Corregido: 14 sep, 2021 | Aceptado: 16 sep, 2021

\section{Introducción}

Desde el año 2010 la Organización Panamericana de la Salud/Organización Mundial de la Salud impulsa la implementación de la estrategia para la Eliminación de la Transmisión Maternoinfantil de la Infección por el VIH, la Sífilis, la enfermedad de Chagas Congénita y la Infección Perinatal por el Virus de la Hepatitis B (ETMI-PLUS), como problemas evitables y de gran impacto en la salud pública.

Las Infecciones de Transmisión Sexual (ITS) se encuentran entre las principales causas de enfermedad en el mundo. Entre ellas, la sífilis es una de las más frecuentes y ha demostrado un aumento de la incidencia en la última década en la Argentina, especialmente en partidos del Conurbano Bonaerense (Ministerio de Salud de la Prov de Buenos Aires, 2019) (Pineda-Leguízamo \& Villasis-Keever, 2017). Su prevalencia suele estar relacionada con la de otras enfermedades de transmisión sexual, lo cual alerta sobre la necesidad de control y prevención del VIH y la hepatitis B, por ejemplo.

Por su parte, la transmisión congénita de la enfermedad de Chagas constituye una importante preocupación en poblaciones como la de la provincia de Buenos Aires (receptora de migrantes externos e internos), ya que, todos los años se registran casos de transmisión vertical de este padecimiento, cuyo diagnóstico y tratamiento temprano se asocia a una muy alta probabilidad de cura.

El Municipio de Almirante Brown (MAB), que forma parte del segundo cordón de los partidos del Conurbano Bonaerense, a través de su Secretaría de Salud, ha orientado sus acciones sanitarias con una fuerte impronta en las acciones preventivas, en particular, ha priorizado la salud materno-infantil como uno de los pilares del sistema de salud. 
Asimismo, la Fundación Mundo Sano (FMS) hace más de veinte años trabaja en disminuir el impacto ejercido por las enfermedades desatendidas en las poblaciones más vulnerables. Sobre todo, eliminar la transmisión materno-infantil de la enfermedad de Chagas $(E C H)$ ha sido una de sus acciones sustantivas. Para ello, ha desarrollado un sinnúmero de acciones que abarcan desde la mejora de la gestión clínica en diversos municipios del país hasta la promoción del desarrollo de uno de los fármacos parasiticidas, el benznidazol, en el marco de una asociación público-privada.

En este contexto y dada la coincidencia de objetivos, ambas instituciones firmaron un acuerdo marco el 28 de marzo de 2016 para trabajar de manera conjunta en la implementación de la estrategia ETMI-PLUS en el Municipio de Almirante Brown, mediante el proyecto MAB-FMS. Los objetivos generales planteados en el acuerdo fueron los siguientes:

I.Implementar la plataforma de eliminación de enfermedades de transmisión materno-infantil ETMI-PLUS en el Municipio de Almirante Brown.

II. Desarrollar un modelo de gestión que pueda ser escalable y reproducible en otros municipios del país.

De dicho modo, las acciones del proyecto MAB-FMS comenzaron en el año de 2018 y se resumen en estos cinco ejes: 1- compromiso político de las autoridades; 2- integración de las cuatro enfermedades en las estrategias para la eliminación de la transmisión vertical y/o tratamiento del recién nacido; 3- integración de los servicios de salud a partir de la creación de una red entre los CAPs del municipio (centros de atención primaria) y los hospitales provinciales de mayor complejidad localizados en el MAB; 4- fortalecimiento de los laboratorios y mejora en la gestión de la cadena de suministros (esta acción coincide plenamente con la Línea de Acción 3 de la propia guía de OPS/OMS de 2017); 5- creación de un nuevo sistema para mejorar los registros tanto de las madres diagnosticadas con alguna de las cuatro enfermedades como de la transmisión congénita a sus hijos.

Aunque existen estudios que analizan el costo efectividad o el costo beneficio del diagnóstico y tratamiento temprano para eliminar la transmisión congénita de cada enfermedad por separado, de momento no se conocen trabajos que analicen en conjunto los retornos de la inversión tras eliminar la transmisión vertical de las cuatro enfermedades bajo la ETMI-PLUS.

A la fecha de elaboración del presente estudio, se cuenta con los primeros resultados posintervención del proyecto MAB-FMS para el fortalecimiento de la ETMI-PLUS en Almirante Brown; por tanto, se busca evaluar sus efectos en términos del Retorno Social de la Inversión, durante su primer año de desarrollo. 


\section{Referente teórico}

Existen antecedentes que dan cuenta de manera individual de la conveniencia económica de implementar medidas para detectar y tratar tempranamente las enfermedades incluidas en la estrategia ETMI-PLUS, con base en análisis de costo-efectividad, eficacia o beneficio.

En el marco de referencia para la eliminación de la sífilis congénita en América Latina y el Caribe (Valderrama, 2005), se citó un informe del Banco Mundial (1993) sobre el desarrollo mundial, el cual estableció que la detección y el tratamiento prenatal de la sífilis es una de las intervenciones disponibles más costo-efectivas. Por ejemplo, en varios estudios y una población teórica del África subsahariana, el costo estimado por caso evitado de sífilis congénita (nacido muerto, bajo peso y otros resultados adversos de la enfermedad) varió entre 44 y 318 dólares, mientras tanto, el costo por AVAD (años de vida ajustados por discapacidad) fue entre 4 y 18,7 dólares.

El estudio de Hong et al. para China (2010) mostró que, además de costo-efectiva, la prevención de la sífilis congénita tiene una relación beneficio-costo elevada de 21,76 . Los autores arribaron a la conclusión de que la detección de la sífilis prenatal combinada con la intervención durante la gestación es muy eficaz.

De otra parte, Ishikawa et al. (2016) evaluaron el coste-eficacia de los enfoques de pruebas de VIH universales y específicas para las mujeres embarazadas en entornos de alta y muy baja prevalencia del virus. En conclusión, el enfoque universal de las pruebas prenatales del VIH logró los mejores resultados sanitarios y ahorró costos (eficaz en función de los costos) a largo plazo en toda la gama de escenarios de prevalencia del VIH. Además, según los autores, las pruebas prenatales de VIH son requisito para una atención sanitaria materno-infantil de calidad y para eliminar la transmisión del virus de madre a hijo.

Otro estudio reciente (Perez-Zetune et al., 2020) valoró los costos de la detección materna, las pruebas y el tratamiento de los lactantes para la enfermedad de Chagas en los Estados Unidos y los comparó con los costos sociales de por vida sin pruebas y las consiguientes morbilidad y mortalidad por falta de tratamiento o tratamiento tardío. Como resultado, se obtuvo un ahorro por nacimiento de 1314 dólares en un programa de detección selectiva y de 105 dólares con la detección universal. Así, se estimó que la evaluación universal genera un ahorro de 420 millones de dólares de por vida, por cohorte de años de nacimiento.

Es importante destacar que la magnitud de la relación entre los costos y la efectividad/beneficios/retornos depende de la situación epidemiológica específica de cada población y de la estructura de costos de las prácticas y los medicamentos. Cuanto mayor sean las prevalencias y las tasas de transmisión vertical, mayores serán los efectos/beneficios/retornos dada una determinada inversión en el diagnóstico temprano de las madres embarazadas, siempre y cuando los costos de los tratamientos tempranos no superen la efectividad/beneficio/retorno de los casos y las complicaciones evitadas, como sugieren los estudios mencionados. En este sentido, el análisis realizado para una población del conurbano bonaerense también resulta novedoso y relevante, pues, está especialmente 
expuesta con un aumento de la incidencia de sífilis en los partidos del Conurbano Bonaerense (Ministerio de Salud de la Provincia de Buenos Aires, 2019) (Pineda-Leguízamo \& Villasis-Keever, 2017).

En este estudio se utiliza el enfoque del Retorno Social de la Inversión (RSI), el cual resulta precisamente útil cuando se busca evaluar las ganancias/pérdidas de un proyecto en términos más amplios que los resultados financieros individuales o privados. Si bien, la metodología fue desarrollada en un inicio para cuantificar el impacto social de la actividad de una empresa u organización, empezó a ser muy provechosa en el ámbito de la salud pública al ayudar a los decisores a concientizar sobre el retorno social de la implementación de acciones concretas, entre ellas, las relacionadas con medidas preventivas (Ivanova, Merino, Gonzalez , \& Jimenez, 2017). Esta tiene por objetivo cuantificar mediante una medida sintética, como es el ratio de retorno/inversión, las ganancias/pérdidas para el conjunto de la sociedad (no solo individuales) a partir de incluir medidas amplias más allá de las erogaciones e ingresos corrientes individuales $y$, en muchos casos, implica combinar medidas cuantitativas, cualitativas, financieras, de costo de oportunidad y/o de gastos evitados.

\section{Métodos}

\subsection{Enfoque}

La investigación es de tipo cuantitativa con un diseño exploratorio y descriptivo.

\subsection{Población del estudio}

El universo objeto de la intervención fueron las mujeres embarazadas que iniciaron sus controles de laboratorio en el Centro Municipal de Diagnóstico (CMD) de Almirante Brown. En 2018, dicho universo fue de 2407 mujeres embarazadas y representó el 47 \% de las mujeres que iniciaron sus controles de laboratorio prenatales en los Centros de Atención de la Salud pertenecientes a dicho municipio ese año.

\subsection{Técnicas de recolección}

La información utilizada provino de a) información primaria de la propia Secretaría de Salud del MAB, en torno a casos diagnosticados (madres embarazadas y niños), resultados positivos de cada enfermedad, tratamientos administrados y seguimiento para la línea de base y para los periodos de implementación del proyecto; b) las tasas de transmisión congénita de cada enfermedad notificadas al Sistema Nacional de Vigilancia de Salud (SNVS) (Ministerio de Salud de la Nación Argentina, s.f.); c) los presupuestos detallados por la FMS respecto a los recursos asignados al proyecto, así como datos fundamentales relacionados con las acciones impulsadas en tal marco y d) los costos de tratamientos e insumos de nomencladores oficiales, a saber: Alfabeta (ALFABETA, s.f.) y el Nomenclador de Hospitales Públicos de Gestión Descentralizada (New LiciSalud, s.f.).

En todos los casos se toma como línea de base el año 2017 para comparar con el 2018, debido a que este último fue el primero donde el MAB trabajó con la FMS en la estrategia para mejorar la puesta en 
práctica de la ETMI-PLUS y del cual se cuenta con la información anual completa al momento de este estudio.

\subsection{Procesamiento del análisis}

A efectos de este trabajo, se aproxima el Retorno a partir de los gastos evitados para el sistema público de salud (denominado ahorros para el sistema) como consecuencia de los resultados atribuibles al proyecto MAB-FMS en términos de los casos y las complicaciones evitadas.

$$
\text { Ratio RI }=\frac{\text { Retorno }}{\text { Inversión }}=\frac{\text { Retorno cuantificable }(\text { ahorros para el sistema de salud })}{\text { Inversión }(\text { erogaciones implementación Proyecto } M A B-F M S)}
$$

De esa forma, la cuantificación del Ratio RI permite conocer cuántos pesos se ahorra el sistema de salud por cada peso invertido, tanto por parte de la FMS como del propio sistema de salud para el diagnóstico, el tratamiento y el seguimiento de las mujeres embarazadas y de los niños objetos de la intervención.

Específicamente, se cuantifican dos tipos de ratios: el primero calculado de modo individual para cada enfermedad y el segundo, de manera agregada para las cuatro enfermedades.

Cabe destacar que, dentro del cálculo de los retornos bajo la metodología del RSI, se pueden incluir otras dimensiones adicionales a los ahorros de recursos para el sistema de salud, por ejemplo: las mejoras en la salud funcional, la calidad de vida, la productividad, la escolaridad, la sobrevida, entre otros. Sin embargo, la valorización monetaria de cada una de estas dimensiones requeriría de datos adicionales indisponibles, por tanto, excede las posibilidades del presente estudio. En esa línea, los resultados obtenidos deben considerarse conservadores, pues, en todos los casos la intervención implica, en alguna medida, mejoras incuantificables con la información existente a la fecha.

Los resultados epidemiológicos sobre los que se realiza la valoración de los retornos, así como los costos que componen la inversión, difieren según las particularidades de cada enfermedad, tal como se explica a continuación:

a) En el caso de sífilis, los retornos provienen del número de casos evitados de niños con sífilis congénita en 2018. Por tanto, el RI en este particular se define como:

$$
R I_{\text {Sífilis }}=\frac{(Q t * C T s)}{\text { Inversión en Sífilis }}
$$

Donde Qt es el número de casos evitados de niños con sífilis congénita en 2018. Dicho valor se calcula como el número de niños nacidos vivos en 2018 por el diferencial de tasa de transmisión de la enfermedad entre el año base 2017 previo a la intervención y la tasa de transmisión luego del primer año de intervención (2018). Es decir, los casos evitados se basan en el contrafactual de cuántos niños vivos habrían contraído sífilis congénita en 2018 si el riesgo de transmisión se hubiese mantenido en los niveles previos a la intervención y cuántos efectivamente nacieron dadas las tasas de transmisión luego de esta última. 
Valga aclarar que, una mejora lograda con la intervención fue la calidad de los registros de laboratorio (diagnóstico de las madres y de los niños); ello se tradujo en la mayor precisión de las tasas de transmisión calculadas a partir de 2018. Sin embargo, a fin de cuantificar el cambio en dichas tasas entre 2017 y 2018, se toma la información proveniente del sistema de vigilancia de la salud (SNVS), que hasta este año no introduce las mejoras en los registros $y$, por ende, contiene un subregistro sistemático (que se asume es aproximadamente el mismo). Dicho cambio porcentual es aplicado a la tasa de transmisión reestimada para 2018, contemplando la mejora en los registros posintervención y, de forma tal, se aproxima mejor el número total de casos de transmisión evitados en este primer año.

Luego, CTs es el costo unitario correspondiente al tratamiento y seguimiento de un niño que por definición nace con sífilis congénita. Dicho valor se obtiene mediante microcosteo, de acuerdo con los protocolos recomendados en las guías oficiales correspondientes (Ministerio de Salud de la Nación, 2016) (Ministerio de Salud de la Prov de Buenos Aires, 2019) (Organización Panamericana de la Salud, Organización Mundial de la Salud, 2017) y los costos unitarios sobre la base de nomencladores oficiales (ALFABETA, s.f.) (New LiciSalud, s.f.).

Así, lo invertido en la intervención de sífilis se compuso de los recursos aportados por la FMS y los del propio sistema de salud del MAB para prevenir de la transmisión vertical de la enfermedad conforme a los protocolos oficiales. La inversión total abarcó los recursos para el diagnóstico en mujeres embarazadas y para el tratamiento y el seguimiento de mujeres embarazadas con dicho padecimiento. El monto total fue calculado mediante microcosteo de las prácticas y los medicamentos descritos en las guías oficiales (Ministerio de Salud de la Nación, 2016) (Organización Panamericana de la Salud, Organización Mundial de la Salud, 2017) y los precios provistos por los nomencladores citados (ALFABETA, s.f.) (New LiciSalud, s.f.).

b) En el caso de la enfermedad de Chagas, la estrategia (ETMI-PLUS) consistió en el tratamiento posparto de las mujeres embarazadas (no es posible tratar a las mujeres durante el periodo de gestación ni de lactancia). Los resultados epidemiológicos considerados para el cálculo de los retornos fueron los siguientes:

I.Los casos adicionales de niños detectados y curados a partir de la intervención (aproximado a partir de las tasas de tratamiento y cura de los recién nacidos en 2018 y 2017).

II.Los casos de nacidos vivos futuros de las madres tratadas gracias a la intervención luego del parto, para quienes se redujo el riesgo de transmisión vertical de la enfermedad.

III.Los casos de madres tratadas posparto en estadio cero de Kuschnir y para las cuales se redujeron sensiblemente todas las complicaciones cardiacas relacionadas con estadios más avanzados de la enfermedad.

Por lo tanto, el RI en el caso de la intervención de la ETMI-PLUS para el caso de chagas se define de esta manera:

$$
R I_{\text {Chagas }}=\frac{[(Q t * C t * E x)+(Q t f * C t f)+(Q t m * C t m * E m x)]}{\text { Inversión en Chagas }}
$$


Donde Qt es el número de casos de niños con chagas congénito curados en 2018 (adicionales desde la intervención). Ct son los costos evitados al sistema, derivados de los tratamientos de las complicaciones asociadas a la enfermedad, que habitualmente ocurren a edades adultas y en su gran mayoría responden a afecciones cardiacas en la Argentina (Lee, Bacon , \& Elen, 2013).

Qtf es el número de casos futuros evitados de niños con chagas congénito (adicionales desde la intervención). La cantidad en 2018 se calculó multiplicando la cantidad de madres diagnosticadas y tratadas en 2018 por el promedio de hijos por mujer $(\mathrm{H})$, al que se le resta el nacimiento actual ponderado por la tasa de transmisión de la enfermedad de Chagas (TC).

Ctf son los costos evitados para el sistema, en este caso, el diagnóstico, el tratamiento temprano y el seguimiento del niño. Qtm, la cantidad de madres diagnosticadas en 2018 y tratadas luego del parto. Ctm, los costos por complicaciones evitadas de esas madres (afecciones cardiacas) y Emx, su esperanza de vida.

Las complicaciones más habituales vinculadas a la enfermedad de Chagas son las afecciones cardiacas con grados variables de complejidad y severidad (Lee, Bacon , \& Elen, 2013). Respecto a este punto, en este trabajo asumimos que aproximadamente el $35 \%$ de los casos permanecen en el estadio 0 de la enfermedad y el $65 \%$ restante evoluciona empeorando progresivamente. Según la literatura clásica, entre el $30 \%$ y el $40 \%$ de los pacientes presenta afectación cardiaca; no obstante, los recientes estudios realizados con resonancia magnética cardiaca han demostrado en estadios iniciales de la enfermedad la presencia de fibrosis y otras alteraciones miocárdicas. Basados en esto, hemos decidido considerar un porcentaje mayor de personas que evolucionan de manera progresiva a otros estadios (Duran-Crane, Rojas, Cooper, \& Medina, 2020).

Dado que no es posible conocer la trayectoria precisa del último grupo (porque es lo evitado gracias al tratamiento oportuno con la intervención), la valoración de los retornos en este caso se basó en tres escenarios hipotéticos construidos sobre la evidencia empírica y la opinión de expertos acerca del tema. Para tal propósito, se tomó en cuenta la edad promedio de las cinco mujeres tratadas en 2018, que era de 33 años al momento del diagnóstico. A continuación, se describen dichos escenarios:

Escenario optimista (E1): es el más optimista en términos de la progresión de las complicaciones, en caso de que las mujeres no reciban tratamiento. Supone lo siguiente: i. El 35 \% (2 mujeres) se mantiene en el estadio 0. ii. El 35 \% (2 casos) empeora, pero solo hasta el estadio 1 (a los 46 años). iii. El 30 \% (1 caso) empeora hasta el estadio 1 (a los 46 años) y luego sigue empeorando hasta el estadio 2 (a los 55 años). En este escenario, se asume para los estadios 0 y 1 de la enfermedad de Chagas una esperanza de vida residual de 37 años adicionales (es decir, viven hasta los 70 años), lo cual implica una reducción de la sobrevida de unos 10 años respecto a la población general de la provincia. En el caso de mujeres que avanzan hacia el estadio 2, se presume el fallecimiento a los 68 años.

Escenario intermedio (E2): es probable e intermedio en cuanto a la gravedad de la evolución de los tres casos que empeoran. Presupone los siguientes aspectos: i. Dos mujeres permanecen en el estadio 0 hasta los 45 años, pasan al estadio I a los 46 años (hasta los 55 años), luego al II a los 56 años y fallecen a los 68 años. ii. Una mujer pasa al estadio I a los 46 años, al II a los 56 años, al III a partir de los 60 años y 
fallece a los 65 años. iii. Como antes, el 35 \% (2 mujeres) se mantienen en el estadio 0, hasta que fallecen a los 70 años.

Escenario pesimista (E3): en el escenario más pesimista, se mantiene a dos mujeres en el estadio 0 hasta el fallecimiento. En el supuesto de quienes empeoran, se considera lo siguiente: i. Una mujer requiere un marcapasos con desfibrilador desde los 46 años y vive hasta los 60 . ii. Una mujer pasa a estadio I a los 46 años, al II a los 56 años, al III a partir de los 60 años y fallece a los 65 años. iii. Una mujer muere a los 40 años de muerte súbita (hasta esa edad estaba en el estadio 0). iv. Como antes, el 35 \% (2 mujeres) se mantiene en el estadio 0 hasta fallecer a los 70 años.

Además, puesto que el tratamiento de las mujeres también reduce la probabilidad del contagio vertical de nuevos embarazos, se plantean dos escenarios alternativos para cada uno de los anteriores: uno en el que se evita un nuevo caso futuro (lo cual implica un mayor retorno por los costos evitados del tratamiento al recién nacido) y otro donde no hay nuevos nacimientos de esas madres o, si los hay, no hay transmisión vertical aún sin el tratamiento de la madre, por cuanto la tasa de transmisión vertical es baja (Danesi , Olenka , \& Sosa-Estani , 2019).

La inversión en chagas abordó los costos de diagnóstico de las embarazadas objeto de la intervención y el costo de tratamiento (posparto y poslactancia) de las mujeres diagnosticadas con la enfermedad y susceptibles de ser tratadas, quienes siguieron el tratamiento con benznidazol (5 en total), en apego al protocolo recomendado (Ministerio de Salud. Presidencia de la Nación. Argentina, 2012).

c) En torno a la hepatitis B (VHB), se registró un aumento en la detección de los casos de mujeres embarazadas con la enfermedad (en 2018 respecto a 2017) atribuible a las mejoras en la etapa diagnóstica desde la implementación del proyecto MAB-FMS.

El incremento en las embarazadas positivas fue de dos casos. La tasa de transmisión vertical de la enfermedad fue entre el 70 \% y el 90 \% (Organización Mundial de la Salud, Hepatitis B, 2020). Al aplicar una tasa promedio del $80 \%$, se estima que los casos evitados de niños nacidos vivos gracias a la detección y al tratamiento oportuno de las madres embarazadas con VHB, habrían sido entre 1 o 2 casos (se aplica 1,6 como proxy de la probabilidad promedio de los casos evitados).

Entonces, el RI en el caso de la intervención de la ETMI-PLUS para el VHB, se define como:

$$
R I_{H B}=\frac{[(Q t * C t)]}{\text { Inversión en } H B}
$$

Donde Qt es el número de casos evitados de niños con HB. Dicho valor se calculó a partir de las dos mujeres reactivas diagnosticadas adicionales en 2018 frente a 2017, considerando una tasa de transmisión vertical de VHB en promedio de 80 \% (Organización Mundial de la Salud, Hepatitis B, 2020).

Ct son los costos evitados para el sistema, en este particular, se asocian con los tratamientos y seguimientos de una persona que padece una infección crónica de VHB.

Dicho valor se obtiene mediante microcosteo, según la opinión de expertos y los costos unitarios sobre la base de nomencladores oficiales (ALFABETA, s.f.) (New LiciSalud, s.f.). 
La inversión para VHB contempló los recursos aportados por la FMS y los provistos por el propio sistema de salud del MAB para prevenir la transmisión vertical de la enfermedad conforme a los protocolos oficiales. La inversión total en prevención se compuso de los recursos para el diagnóstico de la enfermedad de VHB en mujeres embarazadas y para el tratamiento tanto de mujeres embarazadas con VHB como de sus recién nacidos. El monto total fue calculado mediante microcosteo de las prácticas y los medicamentos descriptos en las guías oficiales (Ministerio de Salud de la Nación, 2016) (Organización Panamericana de la Salud, Organización Mundial de la Salud, 2017) y los precios establecidos por los nomencladores aludidos (ALFABETA, s.f.) (New LiciSalud, s.f.).

d) En lo referente al $\mathrm{VIH}$, el incremento en la detección y el tratamiento oportuno de mujeres embarazadas positivas fue de cinco casos (en 2018 respecto a 2017). La tasa de transmisión vertical promedio ascendió al 30 \% (Salud), por tanto, se estima que el número de casos evitados de nacidos con la enfermedad en 2018 habría ascendido a 1 o 2 (1,5 como proxy de la probabilidad promedio de los casos evitados).

Por ende, el RI en el caso de la intervención de la ETMI-PLUS para el VIH, se define como:

$$
R I_{V I H}=\frac{[(Q t * C t)]}{\text { Inversión en VIH }}
$$

Donde Qt es el número de casos evitados de niños con VIH. Dicho valor se calculó a partir de las cinco mujeres reactivas diagnosticadas adicionales en 2018 en comparación con 2017, considerando una tasa de transmisión vertical en promedio de $30 \%$ (Salud).

Ct son los costos futuros evitados para el sistema; estos se asocian con los tratamientos y seguimientos de una persona que padece VIH crónico, con una esperanza considerada de vida de 60 años. Dicho valor se obtiene mediante microcosteo de acuerdo con opinión de expertos y los costos unitarios sobre la base de nomencladores oficiales (ALFABETA, s.f.) (New LiciSalud, s.f.) .

La inversión para VIH fue constituida por los recursos provenientes de la FMS y los provistos por el propio sistema de salud del MAB para prevenir la transmisión vertical de la enfermedad de acuerdo con los protocolos oficiales. La inversión total en prevención abarcó los recursos para el diagnóstico de VIH en mujeres embarazadas, el tratamiento y el seguimiento de mujeres embarazadas que padecen VIH hasta y durante el momento del parto, y el tratamiento y el seguimiento del recién nacido de dichas mujeres. El monto total fue calculado mediante microcosteo de las prácticas y los medicamentos descriptos en las guías oficiales (Ministerio de Salud de la Nación, 2016) (Organización Panamericana de la Salud, Organización Mundial de la Salud, 2017) y los precios facilitados por los nomencladores señalados (ALFABETA, s.f.) (New LiciSalud, s.f.). 


\section{Resultados}

Como se expuso en el apartado anterior, el universo de la intervención fueron las mujeres embarazadas que comenzaron sus controles de laboratorio en 2018 en el Centro de Diagnóstico Municipal del MAB, cuyo número ascendió a 2407. A todas se les realizaron las pruebas diagnósticas para las cuatro enfermedades. De ellas, 226 fueron diagnosticadas con sífilis, 16 con chagas, 8 con VIH y 2 con VHB, a la luz de la información primaria obtenida de la Secretaría de Salud del MAB.

El número de recién nacidos vivos (de las mujeres estudiadas) diagnosticados y tratados con sífilis en 2018 alcanzó los 82 casos y no se registraron nacidos vivos con enfermedad de Chagas, VIH ni VHB. En cuanto a VIH y VHB, no se registraron casos congénitos en el año base 2017 (en los años anteriores al proyecto existían dificultades en las etapas de diagnóstico y registro, por lo cual, resulta imposible asegurar la ausencia de casos no registrados).

En lo concerniente a la enfermedad de Chagas, si bien no se registraron casos congénitos de la enfermedad en 2018, se dieron retornos para el sistema de salud asociados a las otras dos posibles fuentes descritas en el apartado metodológico: la reducción de las complicaciones cardiacas de las madres diagnosticadas y tratadas durante la intervención y el probable decrecimiento de los nacidos vivos futuros con la enfermedad de las madres tratadas, ambas se consideraron en los escenarios utilizados para los cálculos que se presentan a continuación.

A su vez, la estimación de los casos evitados de transmisión vertical de sífilis entre 2017 y 2018 se basó en el contrafactual de cuántos niños habrían nacido con la enfermedad si la tasa de transmisión se hubiera mantenido en los niveles preintervención y la diferencia respecto a lo efectivamente observado (los 82 casos). Para ello, se utilizó la variación en la Prevalencia de Sífilis Congénita en el MAB entre 2017, de acuerdo con la información del Sistema Nacional de Vigilancia de la Salud provista por la Secretaría de Salud del MAB. Según esta, en 2017 la Prevalencia de Sífilis Congénita x 1000 nacidos vivos habría alcanzado a 4,11 y se redujo a 1,95 en 2018. Sobre tal base, los nacidos vivos con sífilis congénita en 2018 habrían ascendido a 173 (si la prevalencia se hubiera mantenido en los niveles de 2017), lo cual representaría una reducción de 91 casos, cifra utilizada como casos evitados.

Entre tanto, de las 16 mujeres diagnosticadas con chagas en 2018, la mitad se encontraba en estadio 0 de Kuschnir (y eran elegibles para ser tratadas), de las cuales cinco efectivamente aceptaron y siguieron el tratamiento con benznidazol. Un trabajo reciente (publicado en 2019) realizó un relevamiento de la información disponible sobre infección congénita por T. cruzi, su evolución y relación con indicadores de prevalencia materna y riesgo vectorial por regiones de Argentina; así, la tasa de transmisión congénita tuvo una evolución irregular durante el período estudiado (2002-2014), con una tasa media nacional que osciló entre el 1,9\% y el 8.2 \% (Danesi , Olenka , \& Sosa-Estani ,2019). A raíz de la baja tasa de transmisión vertical para los retornos, se consideran dos escenarios: un caso futuro evitado de niño vivo con chagas y ningún caso, los que se combinan con los tres escenarios de complicaciones cardiacas de las madres si no hubieran sido tratadas, dando como resultado seis escenarios posibles sobre los cuales se estiman los retornos. 
Para el VIH y el VHB, los casos de transmisión vertical evitados a partir de la intervención ascienden a 1 o 2 para cada una de las enfermedades, de conformidad con lo detallado en el apartado anterior.

En la tabla 1 se presentan los resultados de los retornos (ahorros para el sistema de salud), los costos de la inversión a 2018 y los ratios retorno/inversión para sífilis, chagas, VIH y VHB y para la inversión en las cuatro enfermedades conjuntamente.

\section{Tabla 1}

Retorno, inversión y ratio retorno/inversión del proyecto MAB-FMS

\section{Resultados para sífilis, VIH, hepatitis B, chagas y ETMI-PLUS. Valores en pesos argentinos de 2018.}

\begin{tabular}{|c|c|c|c|c|}
\hline Enfermedad & Escenario & Retorno & Inversión & $\begin{array}{l}\text { Ratio } \\
\text { retorno/inversión }\end{array}$ \\
\hline SÍFILIS ${ }^{(1)(2)}$ & Único & $\$ 2.974 .639$ & $\$ 1.301 .333$ & 2,29 \\
\hline VIH ${ }^{(1)(3)}$ & Único & $\$ 13.847 .365$ & $\$ 2.185 .480$ & 6,34 \\
\hline$H B^{(1)(4)}$ & Único & $\$ 89.780$ & $\$ 713.393$ & 0,13 \\
\hline \multirow{6}{*}{$\underset{\text { (1) (5) }}{\text { CHAGAS }}$} & Optimista + Ningún caso futuro & $\$ 504.154,58$ & $\$ 383.317,08$ & 1,32 \\
\hline & Intermedio + Ningún caso futuro & $\$ 1.175 .978,82$ & $\$ 383.317,08$ & 3,07 \\
\hline & Pesimista + Ningún caso futuro & $\$ 1.102 .026,16$ & $\$ 383.317,08$ & 2,87 \\
\hline & Optimista + Un caso futuro & $\$ 505.884,04$ & $\$ 383.317,08$ & 1,32 \\
\hline & Intermedio + Un caso futuro & $\$ 1.177 .708,29$ & $\$ 383.317,08$ & 3,07 \\
\hline & Pesimista + Un caso futuro & $\$ 1.103 .755,63$ & $\$ 383.317,08$ & 2,88 \\
\hline \multirow{6}{*}{ ETMI-PLUS ${ }^{(6)}$} & Optimista + Ningún caso futuro & \$17.415.938 & $\$ 4.583 .524$ & 3,80 \\
\hline & Intermedio + Ningún caso futuro & $\$ 18.087 .763$ & $\$ 4.583 .524$ & 3,95 \\
\hline & Pesimista + Ningún caso futuro & $\$ 18.013 .810$ & $\$ 4.583 .524$ & 3,93 \\
\hline & Optimista + Un caso futuro & $\$ 17.417 .668$ & $\$ 4.583 .524$ & 3,80 \\
\hline & Intermedio + Un caso futuro & $\$ 18.089 .492$ & $\$ 4.583 .524$ & 3,95 \\
\hline & Pesimista + Un caso futuro & $\$ 18.015 .539$ & $\$ 4.583 .524$ & 3,93 \\
\hline
\end{tabular}

Fuentes: Secretaría de Salud del Municipio de Almirante Brown; Sistema Nacional de Vigilancia Epidemiológica; Ministerio de Salud de la Nación; Fundación Mundo Sano; Grupo Alfabeta (Alfabeta.net); New LiciSalud (Nomencladores).

Notas:

(1)- Inversión estimada sobre la base del diagnóstico, seguimiento y tratamiento correspondiente, según la enfermedad, de 2407 mujeres embarazadas en 2018 que iniciaron sus controles en el Centro de Diagnóstico Municipal de Almirante Brown.

(2)- Retorno estimado sobre la base de 91 casos evitados de transmisión vertical de sífilis.

(3)- Retorno estimado sobre la base de 1 o 2 casos evitados de transmisión vertical de $\mathrm{VIH}$ (1,5 como probabilidad promedio, dada la tasa de transmisión).

(4)- Retorno estimado sobre la base de 1 o 2 casos evitados de transmisión vertical de HB (1,6 como probabilidad promedio, dada la tasa de transmisión).

(5)- Retorno estimado sobre la base de los escenarios de evolución de complicaciones cardiacas evitadas entre las 5 las mujeres que recibieron tratamiento y uno o ningún caso evitado de transmisión futura de esas 5 mujeres.

(6)- Retorno e inversión globales para ETMI-PLUS = la suma de los retornos y las inversiones de las 4 enfermedades, respectivamente. 
Los resultados de sífilis muestran un ahorro para el sistema de salud (retorno) de casi 3 millones de pesos (a precios constantes de 2018), como consecuencia de los costos asociados a tratamientos de los 91 casos de transmisión vertical que se estima fueron evitados a partir de la implementación del proyecto MAB-FMS (costos conformados por estudios de laboratorio, tratamiento y seguimiento de los niños nacidos con dicha enfermedad y de los honorarios y los viáticos adicionales aportados por el proyecto). Por su parte, la inversión asociada a este resultado habría ascendido a 1,3 millones de pesos (de 2018) e incluye los recursos aportados tanto por la FMS como por el propio sistema de salud para el diagnóstico de las 2407 mujeres embarazadas (reactivas y no reactivas) y el costo de tratamiento y seguimiento (durante el parto y el posparto) de aquellas cuyo resultado final fue reactivo, de acuerdo con lo indicado en las guías oficiales citadas oportunamente. Así, la relación ratio retorno de la inversión señala que, por cada peso invertido en el proyecto MAB-FMS para la eliminación vertical de sífilis, el sistema de salud ahorra 2,29 pesos en tratamiento y seguimiento de los niños nacidos vivos con dicho padecimiento.

En torno al VIH, los retornos ligados a la eliminación vertical de nacidos vivos (1 o 2) con la enfermedad ascenderían, en promedio, a casi 14 millones de pesos (a precios constantes del 2018) y se relacionan con los ahorros en tratamiento, controles y seguimiento de esos niños nacidos vivos con VIH durante el tiempo que se estima vivirán, en concordancia con la línea de base de los protocolos oficiales. A la fecha, no existen estimaciones empíricas de la esperanza de vida al nacer de una persona con VIH para la Argentina (por la relativamente reciente trayectoria de la enfermedad) y, si bien, la evidencia internacional muestra un aumento de la sobrevida de las personas con VIH, sigue siendo menor a la del promedio de la población (Argañaráz, Alarcón, Genero, Flores Barros, \& Quintana , 2018). Dado lo anterior y según la opinión de expertos, es razonable asumir una esperanza de vida al nacer de 60 años, es decir, 16,5 años menos que el promedio de la población de la provincia, estimada en 76,5 años, de acuerdo con proyecciones propias basadas en el Population Analysis Spreadsheets (PAS) (Arriaga, 2001). Asimismo, la inversión estimada en VIH contempló los costos de las pruebas diagnósticas a las 2047 mujeres embarazadas (reactivas y no reactivas), el tratamiento a las mujeres reactivas durante el embarazo y durante el parto y el tratamiento de los niños nacidos vivos de las madres positivas (más los honorarios y los viáticos adicionales asignados por la intervención), todo lo cual equivalió a 2,2 millones de pesos (de 2018). Por tanto, el ratio retorno/inversión indica que, por cada peso invertido en el proyecto para la eliminación vertical de $\mathrm{VIH}$, el sistema de salud ahorra 6,3 pesos.

De otro lado, los retornos vinculados con la eliminación de la transmisión vertical de 1 o 2 casos de hepatitis B alcanzarían, en promedio, casi los 90 mil pesos (a valores constantes de 2018), tomando en cuenta el costo de tratamiento y seguimiento promedio a los niños nacidos vivos con la enfermedad. La inversión realizada por el proyecto en este caso superó el retorno, pues se elevó a 713 mil pesos (de 2018) compuesto por las pruebas diagnósticas a las 2407 mujeres embarazadas y el tratamiento de las mujeres embarazadas positivas y de sus nacidos vivos (incluidos los honorarios y los viáticos aportados por el proyecto). Entonces, el ratio retorno de la inversión es menor a uno, o sea, el costo de la eliminación vertical supera los ahorros futuros para el sistema de salud por el tratamiento a los niños (nacidos con la enfermedad de madres no tratadas).

Para la enfermedad de Chagas, los retornos estimados varían en un rango de 504 mil pesos a 1,17 
millones de pesos (valores constantes de 2018), dependiendo del escenario de complicaciones cardiacas futuras de las mujeres embarazadas con la enfermedad (sin tratamiento) y de los casos evitados de niños futuros. En todos ellos, dichos retornos superarían la inversión realizada por el proyecto, compuesta por las pruebas diagnósticas para detectar la enfermedad en las 2407 mujeres embarazadas (reactivas y no reactivas), los estudios complementarios a las mujeres reactivas, el diagnóstico y el tratamiento a los recién nacidos de las madres reactivas y el tratamiento posparto a las mujeres con el padecimiento, aunque no es posible el tratamiento durante la gestación (incluidos los honorarios y los viáticos aportados por el proyecto). De ese modo, los ratios retorno/inversión son mayores a 1 en todos los casos entre un rango de 1,3 - 3, lo cual sugiere que, de cada peso invertido en chagas, la intervención generó un ahorro de 1,3 a 3 pesos para el sistema de salud, fundamentalmente gracias a las complicaciones cardiacas evitadas entre las mujeres reactivas que reciben tratamiento oportuno.

Finalmente, el análisis conjunto de la intervención para el fortalecimiento de la ETMI-PLUS en el Municipio de Almirante Brown, se efectuó tomando la totalidad de la inversión en pruebas diagnósticas y tratamientos para la eliminación vertical de las cuatro enfermedades desde la implementación del proyecto y los retornos en términos de las mejoras en los resultados globales respecto al año base o año preproyecto (2017).

Este resultado se obtuvo con la suma de la inversión en cada enfermedad, por un lado, y la suma de los retornos, por el otro. Para tal propósito, se tomaron los resultados de los escenarios únicos para sífilis, $\mathrm{VIH}$ y VHB y los cuatro escenarios para chagas. Consecuentemente, se obtuvo que los retornos o ahorros para el sistema de salud, gracias a las mejoras en la eliminación vertical de las cuatro enfermedades y a las complicaciones cardiacas evitadas en las mujeres con chagas, serían entre 17 y 18 millones de pesos (a valores constantes de 2018), mientras que, la inversión habría sido del orden de 4,5 millones de pesos (también a valores de 2018). Por lo tanto, por cada peso invertido en el fortalecimiento de la ETMI-PLUS en el MAB (tanto por el propio sistema de salud como por la Fundación Mundo Sano), el retorno sería de casi 4.

\section{Conclusiones}

La principal conclusión de este estudio es la existencia de una relación retorno/inversión favorable a la intervención analizada: el fortalecimiento de la ETMI-PLUS en el Municipio de Almirante Brown, Provincia de Buenos Aires, Argentina.

Los resultados mostraron que, por cada peso invertido en el fortalecimiento de la ETMI-PLUS en el MAB por parte de la Fundación Mundo Sano y del sistema de salud en el año 2018, se habría obtenido un retorno de casi 4 pesos, gracias a las mejoras en la eliminación vertical de las cuatro enfermedades (sífilis, $\mathrm{VIH}$, hepatitis B y chagas) y a la reducción de las complicaciones cardiacas en las mujeres embarazadas diagnosticadas con chagas y tratadas oportunamente.

El análisis de los ratios retorno de la inversión para cada una de las enfermedades de manera individual 
evidenció que, para tres de las cuatro enfermedades (sífilis, VIH y chagas), los valores de los retornos superan los montos invertidos en pruebas diagnósticas y tratamientos para la eliminación vertical, en línea con los hallazgos de estudios antecedentes de costo efectividad o costo beneficio para esas enfermedades en otras poblaciones (Hong, y otros, 2010) (Ishikawa, y otros, 2016) (Lee, Bacon , \& Elen, 2013).

A fin de comprender los alcances y las limitaciones de estos resultados, es importante considerar la definición ad-hoc de retorno, condicionada a la información disponible. Así, para la cuantificación de los retornos se incluyen exclusivamente los ahorros para el sistema de salud en tratamientos y seguimiento de casos y complicaciones de enfermedades, gracias a los resultados obtenidos con el proyecto FMS$M A B$; pero, no se incluyen otras dimensiones muy relacionadas con las mejoras en los resultados alcanzados. Los retornos no considerados consisten en aspectos subjetivos (de más difícil cuantificación económica), como las mejoras en la calidad de vida autoperciba por parte de los individuos y sus familias de los casos y las complicaciones evitadas y aspectos más mesurables (en caso de contarse con la información necesaria), a saber, las mejoras en la escolaridad, en la productividad y en la sobrevida de quienes evitaron dichas enfermedades. En este sentido, los resultados del presente estudio pueden considerarse valores conservadores del verdadero retorno social obtenido gracias a la intervención.

Los cálculos de los retornos y de las inversiones en cada una de las enfermedades implicaron una serie de desafíos, que se describen como sigue: i) los precios de las prácticas y de los insumos (incluidos medicamentos) difieren según el proveedor o el demandante, aun para productos idénticos. Uno de los casos más notorios son los medicamentos antiretrovirales para el tratamiento del VIH, cuyos precios de mercado (ALFABETA, s.f.) son muy superiores al de las compras públicas, siendo este último más representativo, ya que el principal proveedor es el Ministerio de Salud de la Nación. Para evitar que la relación ratio retorno de la inversión se vea afectada por dichas diferencias, para cada enfermedad se tomó la misma fuente de información para el cálculo de los retornos y de la inversión, con prioridad en los precios públicos cuando estuvieran disponibles (como en el caso de VIH). Para las otras enfermedades, dado que no se contaba con información precisa del valor de los insumos y los medicamentos vía compras públicas, se consideró en todos los casos los precios de mercado (tanto para el cálculo de los retornos como para los insumos relacionados con la inversión). En cuanto a los valores de las prácticas (internaciones, consultas, etc.), se trabajó con los valores provistos por el Nomenclador para Hospitales Públicos de Gestión Descentralizada (New LiciSalud, s.f.), considerada la fuente de información más representativa del sector; ii) la valorización de los retornos fue la definición de los tratamientos más representativos en cada caso y dos aspectos resultaron especialmente relevantes: 1el hecho de que para muchos casos no existe un único tratamiento disponible; 2- que las enfermedades evitadas en muchas ocasiones se asocian a otras comorbilidades. El criterio en ambos fue considerar los escenarios más conservadores, para los cuales se tomaron, en primer lugar, los tratamientos de línea de base o más habitualmente utilizados en el sector público (de acuerdo con la opinión de expertos en el tema), que además suelen ser los de menor costo, y, en segundo lugar, no se contemplaron los costos de los tratamientos por las comorbilidades asociadas (excepto en el caso de las complicaciones cardiacas evitadas por las madres con chagas que reciben tratamiento oportuno).

Además, es importante resaltar que el estudio busca evaluar los ratios retorno/inversión del proyecto 
MAB-FMS que busca fortalecer la estrategia ETMI-PLUS en el Municipio de Almirante Brown. Los resultados de esta intervención no son generalizables a otras poblaciones porque dependen de los recursos asignados y también, en gran medida, de la situación epidemiológica específica de la población objeto y de la calidad de las acciones implementadas con el proyecto.

Durante la implementación, como ya se mencionó, se pudieron efectuar mejoras en aspectos de meso y microgestión sanitaria (informatización de historias clínicas, manuales de procedimientos en el laboratorio central, capacitación continua del personal, generación de redes de atención y seguimiento de casos a través de la red, etc.) que permitieron los resultados sanitarios logrados por la estrategia. Igualmente, la previsibilidad de los recursos otorgados por FMS permitió la atención ininterrumpida de pacientes e implementar herramientas de mejora continua, lo cual se tradujo en resultados más óptimos y, a la vez, facilitó liberar fondos de la secretaría para ser redirigidos a otras necesidades como infraestructura o bienes de capital, brindando un marco cierto para la planificación de las finanzas públicas.

Finalmente, es importante resaltar que este trabajo ofrece información objetiva sobre aspectos financieros de una asociación público-privada orientada a fortalecer una política pública sanitaria, situación de la cual no se consignan antecedentes. La posibilidad de que una inversión privada libere fondos públicos en contexto de restricción fiscal constituye una herramienta de gran potencialidad social.

\section{Agradecimientos}

Para el desarrollo de este estudio se contó con el apoyo financiero de la Fundación Mundo Sano durante el primer semestre del año 2020, que permitió la participación de la investigadora Constanza Silvestrini, a través de la Fundación Quant. Las autoras agradecen a la Dra. Silvia Gold y al Lic. Marcelo Abril de la Fundación Mundo Sano por promover y apoyar la realización del presente estudio. A la Fundación Quant, por apoyar el desarrollo del presente trabajo y las valiosas sugerencias emitidas para su diseño. Al Dr. Bruno Lococo, cuya amplia experiencia en lo referido a la evolución y al tratamiento de pacientes con enfermedad de Chagas, resultó clave para la aproximación de los costos y los retornos asociados con esta. A los evaluadores y al comité editorial de la revista, por sus valiosos comentarios que ayudaron a mejorar la versión original del manuscrito. 


\section{Referencias}

ALFABETA. (s.f.). https://www.alfabeta.net/home/. Recuperado de http://www.alfabeta.net/precio/

Argañaráz, A. C., Alarcón, A. M., Genero, S., Flores Barros, M. E., \& Quintana , J. C. (2018). Análisis de supervivencia de pacientes con diagnóstico de vih del programa vih-sida, durante el período 1987 - 2017. En F. d.-U. Nordeste, Libro de Artículos Científicos en Salud . Recuperado de https://med.unne.edu.ar/index.php/institucional/cienciaytecnologia/libroarticuloscientificos/

Arriaga, E. (2001). El Análisis de la Población con Microcomputadoras. Córdoba, España: Universidad Nacional de Córdoba. Recuperado de https://books.google.com.ar/books?id=jOWAGwAACAAJ

Danesi , E., Olenka , M., \& Sosa-Estani , S. (2019). Transmisión Congénita de TRYPANOSOMA CRUZI. Argentina 2002-2014. MEDICINA, 79, 81-89.

Duran-Crane, A., Rojas, C., Cooper, L., \& Medina, H. (2020). Cardiac Magnetic Resonance Imaging in Chagas' disease: a parallel with electrophysiologic studies. International journal of cardiovascular imaging, 36(11), 2209-2219. doi: 10.1007/s10554-020-01925-2

Hong, F.-C., Liu, J.-B., Feng, T.-J., Liu, X.-L., Pan, P., Zhou, H., Cai, Y. M., Ling, L., Huang, X. M., Zhang, D., Zhang, Y. J., \& Zeegers, M. P. ( 2010). Congenital Syphilis: An Economic Evaluation of a Prevention Program in China. Sexually Trnsmitted Diseases, 37(1), 26-31. doi: 10.1097/OLQ.0b013e3181b3915b

Ishikawa, N., Dalai, S., Johnson, C., Hogan, D. R., Shimbo, T., Shaffer, N., Pendse, R. N., Lo, Y. R., Ghidinelli, M. N., \& Baggaley, R. (2016). Should HIV testing for all pregnant women continue? Costeffectiveness of universal antenatal testing compared to focused approaches across high to very low HIV prevalence settings. Journal of the International AIDS Society, 19(1), 21212. doi: 10.7448/IAS.19.1.21212

Ivanova, Y., Merino, M., Gonzalez , A., \& Jimenez, M. (2017). Retorno Social de la Inversion en Salud. Revista Española de Economia de la Salud, 12(4), 686-693.

Lee, B., Bacon , K., \& Elen, M. (2013). Global economic burden of Chagas disease: a computational simulation model. The Lancet. Infectious diseases, 13(4), 342-348. doi: 10.1016/S1473-3099(13)70002-1

Ministerio de Salud de la Nación Argentina. (s.f.). Epidemiología y análisis de situación de salud. Recuperado de https://www.argentina.gob.ar/salud/epidemiologia

Ministerio de Salud de la Nación Argentina. (2016). Prevención de la transmisión perinatal de Sífilis, Hepatitis B y HIV. Recomendaciones para el trabajo de los equipos de salud.Recuperado de 
https://bancos.salud.gob.ar/recurso/prevencion-de-la-transmision-perinatal-de-sifilis-hepatitisb-y-vih

Ministerio de Salud de la Provincia de Buenos Aires, Argentina. (2019). Estretegia de intervención para la prevención de la sífilis materna y congénita en la Provincia de Buenos Aires. Recuperado de https://www.ms.gba.gov.ar/sitios/hiv/files/2019/04/Estrategia-de-intervenci\%C3\%B3n-para-laprevenci\%C3\%B3n-de-la-s\%C3\%ADfilis-materna-y-cong\%C3\%A9nita-en-la-Provincia-deBuenos-Aires.pdf

Ministerio de Salud de la Nación Argentina. (2018). Guías para la Atención al Paciente Infectado con Trypanosoma Cruzi (Enrermedad de Chagas). Recuperado de https://bancos.salud.gob.ar/recurso/guias-para-la-atencion-al-paciente-infectado-contrypanosoma-cruzi-enfermedad-de-chagas

New

LiciSalud.

(s.f.).

https://www.obrasociales.com.ar/.

Recuperado

de https://www.obrasociales.com.ar/nomencladores/

Organización Panamericana de la Salud/Organización Mundial de la Salud. (2008). Eliminación mundial de la sífilis congénita: fundamentos y estrategia para la acción. Recuperado de https://www.who.int/reproductivehealth/publications/rtis/9789241595858/es/

Organización Mundial de la Salud. (2019). VIH/SIDA. Recuperado de https://www.who.int/es/newsroom/fact-sheets/detail/hiv-aids

Organización Mundial de la Salud. (2020). Hepatitis B. Recuperado de https://www.who.int/es/newsroom/fact-sheets/detail/hepatitis-b

Organización Panamericana de la Salud. (2005). Eliminación de sífilis congénita en América Latina y el Caribe: marco de referencia para su implementación. Recuperado de http://www.paho.org/clap/index.php?option=\%20com_docman\&task=doc_view\&gid=\%20120 \&ltemid $=247$

Organización Panamericana de la Salud. (2016). Plan de acción para la prevención y el control de la infección por el VIH y las infecciones de transmisión sexual 2016-2021. Recuperado de https://iris.paho.org/handle/10665.2/34079

Organización Panamericana de la Salud, Organización Mundial de la Salud. (Julio, 2017). ETMI-PLUS: marco para la eliminación de la transmisión maternoinfantil del VIH, la síficilis, la hepatitis y la enfermedad de Chagas. Recuperado de https://www.paho.org/hq/dmdocuments/2017/2017-cha-etmi-PLUSmarco-vih-hep-chagas.pdf

Perez-Zetune, V., Bialek, S. R., Montgomery, S. P., \& Stillwaggon, E. (2020). Congenital Chagas Disease in the United States: The Effect of Commercially Priced Benznidazole on Costs and Benefits of Maternal Screening. The American journal of tropical medicine and hygiene, 102(5), 1086-1089. doi: 10.4269/ajtmh.20-0005 
Pineda-Leguízamo, R., \& Villasis-Keever, M. (2017). Sífilis congénita: un problema vigente. Revista Mexicana de Pediatría, 84(2), 45-47. Recuperado de https://www.medigraphic.com/pdfs/pediat/sp2017/sp172a.pdf

Puigbo, J. (2001). Contribución al estudio de la miocardia chagásica crónica en Venezuela. Una visión panorámica. Parte II. Gaceta Médica de Caracas, 109(3), 304-317. 


\section{Población y Salud en Mesoamérica}

¿Quiere publicar en la revista?

Ingrese aquí

O escríbanos:

revista@ccp.ucr.ac.cr

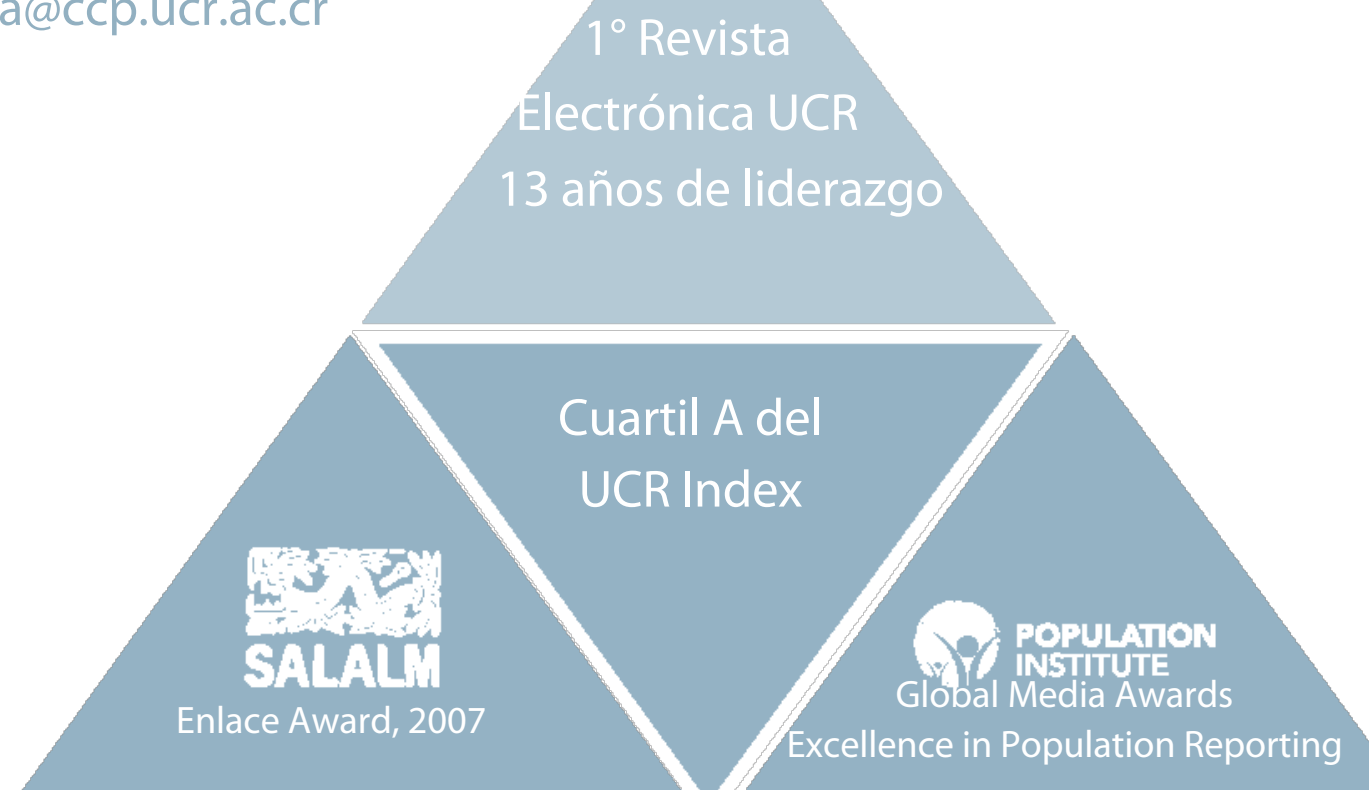

Población y Salud en Mesoamérica (PSM) es la revista electrónica que cambió el paradigma en el área de las publicaciones científicas electrónicas de la UCR. Logros tales como haber sido la primera en obtener sello editorial como revista electrónica la posicionan como una de las más visionarias.

\section{Revista PSM es la letra delta mayúscula, el cambio y el futuro.}

Indexada en los catálogos más prestigiosos. Para conocer la lista completa de índices, ingrese aquí.

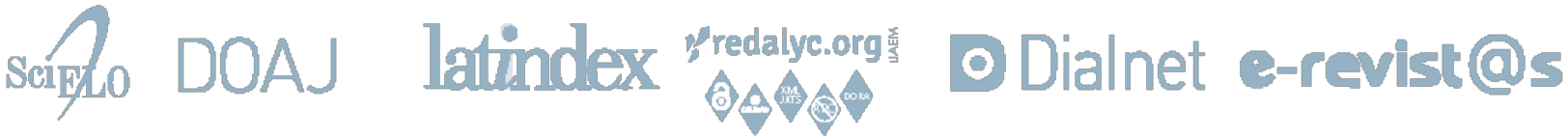

Revista Población y Salud en Mesoamérica - PSM

Centro Centroamericano de Población

Universidad de Costa Rica

\section{(요우}

